\title{
Rate Adaptive AV delay and Exercise Performance following Cardiac Resynchronization Therapy
}

\author{
Nesan Shanmugam, MBBS, MRCP ${ }^{1}$; Oscar Prada-Delgado, MD $^{1}$; \\ Ana Garcia Campos, $\mathrm{MD}^{1 ;}$ Alex Grimster, $\mathrm{BSc}^{1}$; Oswaldo Valencia, $\mathrm{MD}^{1}$; \\ Aigul Baltabaeva, $\mathrm{MD}^{1}$; Sue Jones, $\mathrm{BSc}^{1}$; Lisa Anderson, $\mathrm{MD}^{1}$. \\ ${ }^{1}$ St George's Healthcare NHS Trust, Department of Cardiology, London, \\ United Kingdom.
}

\begin{abstract}
Address for correspondence:
Dr Nesan.Shanmugam, St George's Healthcare NHS Trust, Department of Cardiology, Blackshaw Road, London SW17 0QT, United Kingdom, Email: nesan_s@ @hotmail.com Telephone: (+44) 2087251220 Facsimile: (+44)208725
\end{abstract}

Conflicts of interest: $\mathrm{N}$ Shanmugam has received research funding support from Medtronic and St Jude Medical. 


\begin{abstract}
Background: Physiological shortening of the atrioventricular (AV) interval with increasing heart rate is well documented in normals, and an established component of dual chamber pacing for bradycardia.
\end{abstract}

Objectives: To assess the effect of exercise on optimal AV delay and the impact of a patient specific rate adaptive AV delay on exercise capacity in heart failure (HF) patients following cardiac resynchronization therapy (CRT).

\title{
Methods:
}

Phase 1: We performed iterative AV optimization at rest and exercise in 52 CRT patients in atrial sensed mode (mean age $71.6 \pm 9.2 \mathrm{y}, 25 \%$ female).

Phase 2: Subsequently, 20 consecutive volunteers from this group (mean age 69.2 $\pm 9.6 \mathrm{y}$, $15 \%$ female), underwent cardiopulmonary exercise testing (CPET) with rate adaptive AV delay (RAAVD) individually programmed on (RAAVD-ON) or off (RAAVD-OFF).

\section{Results:}

Phase 1: In 94\%, there was a marked reduction (mean 50\%) in optimal AV delay with exercise. The optimal resting versus exercise AV delay was $114.2 \pm 29 \mathrm{~ms}$ at heart rate (HR) 64.4 $\pm 7.1 \mathrm{bpm}$ versus $57 \pm 31 \mathrm{~ms}$ at $\mathrm{HR} 103 \pm 13 \mathrm{bpm}, \mathrm{p}<0.001$. No patients required an increase in AV delay with exercise and $3(6 \%)$ showed no change.

Phase 2: With RAAVD-ON significantly better exercise times were achieved $(8.7 \pm 3.2$ minutes) compared with RAAVD-OFF (7.9 \pm 3.2 minutes; $\mathrm{p}=0.003)$, and there was a 
significant improvement in VO2max (RAAVD-ON 16.1 \pm 4.0 versus RAAVD-OFF $14.9 \pm 3.7 \mathrm{ml} / \mathrm{kg} / \mathrm{min} ; \mathrm{p}=0.024)$.

Conclusions: There was a dramatic reduction in optimal AV delay with physiological exercise in the majority of this HF CRT cohort. Replicating this physiological response with a programmable rate adaptive AV delay translated into a $10 \%$ improvement in exercise capacity.

\section{Key Words}

Heart failure; Cardiac resynchronization therapy; Cardiopulmonary exercise testing; Atrioventricular Delay Optimization; Rate adaptive pacing; Exercise. 


\section{Introduction}

Following cardiac resynchronization therapy (CRT), the atrioventricular (AV) interval can be individually modified to enhance ventricular filling and preload (AV delay optimisation). The acute hemodynamic benefits of AV optimization are well described in resting patients and suboptimal programming of the AV delay can result in up to a $15 \%$ reduction in cardiac output (1).

Currently, AV delay optimization is performed at rest; however, the patient is likely to require the most favorable filling conditions during exercise. In healthy subjects, an inverse relationship exists between AV interval and heart rate (HR) (2), and in dual chamber pacing for bradycardia, there is some evidence that reduction of AV interval with increasing HR improves hemodynamics $(3,4)$ forming the basis for the 'rate adaptive AV delay' function in contemporary pacemakers.

However, there is conflicting evidence on the effect of increasing heart rate on the optimal AV delay in the CRT population. Employing different optimization methods, and heart rate modulation protocols, it has been reported that the AV delay increases, decreases, or stays the same with increasing heart rate (5-11). Due to these inconsistent findings, it is currently advised that the rate adaptive AV delay function is inactivated (7, $10)$.

Most studies of AV optimization with exercise in CRT have employed echocardiographic left ventricular outflow tract velocity time integral (LVOT-VTI) as a surrogate for stroke 
volume $(5,6,10)$. However, it is left ventricle (LV) diastolic filling waves that are attenuated during exercise and it would appear more intuitive to consider manipulating the LV diastolic filling pattern directly, an approach now well established $(12,13)$ Therefore using the simplified iterative technique recently recommended by the American Society of Echocardiography (14), we examined the effect of exercise on optimal AV delay in CRT patients. On the basis of our findings, we then individually programmed rate adaptive AV delay in a subset of 20 patients, in order to assess the effect on exercise capacity using cardiopulmonary exercise testing (CPET). 


\section{Methods}

\section{Phase 1}

Patient Population. We recruited 52 patients following CRT implantation for clinical indications at St George's Hospital between 2009 and 2010. All patients were required to be more than 3 months post implantation, be clinically stable and to have a sufficient level of fitness and mobility in order to undergo bicycle exercise echocardiography in an upright position and to demonstrate sinus rhythm with atrial sensed (AS) biventricular pacing. Patients were excluded from the study if there was a history of recent myocardial ischemic event within the last 3 months, structural valve disease, or a technically satisfactory echocardiogram could not be obtained at rest.

Study Design. Patients underwent iterative AV optimization at rest and exercise. Pacing was programmed in VDD 50 atrial sensed mode, and the interventricular delay was set at $0 \mathrm{~ms}$. Algorithms for rate response and rate adaptive AV delay were turned off. Patients were instructed to take their medications as usual. The study protocol was approved by the local Research Ethics Committee and all patients provided written informed consent.

\section{Imaging}

The echocardiographic images were recorded with a Vivid 7 ultrasound system (Vingmed Ultrasound AS, General Electric, Horten, Norway) equipped with a 2.5- to 5$\mathrm{MHz}$ imaging probe and off-line cine-loop analysis software. The images were acquired in a semi-supine position, at rest and during upright exercise, and the mean value of three consecutive cardiac cycles was taken for each echocardiographic variable. The images 
were recorded by a single physician and then analyzed once the patient was resting. Reproducibility- The intraobserver and interobserver variability of LV filling time (LVFT) on exercise (expressed by the coefficient of variation (\%)) and the interobserver concordance to within $20 \mathrm{~ms}$ for the optimal exercise AV delay was calculated in 20 patients.

\section{AV Optimization}

At Rest: The iterative method was used for the optimization of the AV delay at rest. (15) This was performed using pulsed-wave Doppler of the trans-mitral flow to detect the longest LVFT without truncation of the A-wave by mitral valve closure at the onset of ventricular systole.

At Peak Exercise: Patients underwent a symptom limited, 'pedalo' modified Tenturi E80 exercise bicycle test. The Tenturi E80 exercise bicycle was modified by replacing the existing seat with a handlebar accessory thereby enabling the patient to safely exercise whilst seated on the edge of the echocardiography couch using the 'pedalo' posture. After an initial 3 minutes at $20 \mathrm{~W}$, the workload was increased by $10 \mathrm{~W}$ every 2 minutes until patients developed symptoms. Images were acquired immediately after peak exercise, whilst the patient remained seated at the bedside. If the heart rate fell quickly, before the optimal filling pattern had been detected, the patient was submaximally exercised for a brief period until peak HR was reachieved to allow the acquisition of further images.

The AV delay was changed until the optimal mitral E and A separation and LVFT on pulsed-wave Doppler was achieved. Blood pressure and 12-lead electrocardiograms 
(ECG) were recorded at 1 minute intervals. 12 lead ECGs were assessed for appropriate biventricular capture throughout the test. Exercise duration and peak HR were recorded.

\section{Phase 2}

All patients taking part in phase 1 were invited to participate in a substudy involving two CPET tests performed one week apart. The first 20 patients to volunteer were included. All patients were randomized in a cross over design to undergo CPET with their CRT device programmed to VDD mode with fixed AV delay (RAAVD-OFF), and VDD mode with rate adaptive AV delay algorithm ON (RAAVD-ON). CPET was performed 7 days apart and the patient and operator supervising the CPET were blinded to the programmed pacing configurations. Three different device models were implanted in the study population (Guidant Contak Renewal TR2 in 12 (60\%) patients; Biotronik Lumax 540 HF-T in 4 (20\%) and Medtronic Concerto II D294TRK in 4 (20\%)) each with their own individual rate adaptive AV delay algorithms (Table 1).

The devices were programmed to a lower rate limit of 50 beats per minute (bpm) and an upper rate limit of $140 \mathrm{bpm}$. For the rate adaptive algorithm, the maximum setting was programmed to equal the optimal AV delay at rest and the minimum setting equaled the optimal AV delay after peak exercise, as measured in phase 1 of the study.

Cardiopulmonary exercise testing. All subjects performed a symptom-limited erect treadmill exercise test (Q5000, Quinton, Seattle, Washington) using an incremental ramp protocol with simultaneous respiratory gas analysis (Cardiopulmonary Exercise Testing System, MedGraphics, St. Paul, Minnesota). (16) The gas analyzer was calibrated with 
standard gases immediately before the test. Sampling of expired gases was performed continuously, and data was expressed as 30-second means. Standard 12-lead ECG and non-invasive blood pressures were recorded every 2 minutes throughout the study. The 12 lead ECGs were assessed throughout the test to check for appropriate biventricular capture without ventricular fusion at longer AV delays. The following variables were determined: peak HR, exercise time, and peak myocardial oxygen consumption (VO2max) which was defined as the average value of VO2max measured during the last 30 seconds of exercise. Exercise was terminated at the subject's request because of fatigue or breathlessness. Patients were instructed to continue with their medications as usual.

\section{Data Analysis.}

Results are presented as mean \pm 1 standard deviation (SD) for continuous normallydistributed variables, as median [interquartile range] for continuous skewed distributed data, and as percentages for categorical data. Analysis of normality was performed with the Kolmogorov-Smirnov and Shapiro-Wilk test.

Paired $t$-test was used to compare values determined at rest and during exercise in phase 1 of study. Comparisons of the results in RAAVD-ON and RAAVD-OFF in phase 2 were analyzed using the paired $t$-test with adjustment for period effects. The magnitude of change was estimated with the $95 \%$ confidence interval. Linear regression analysis was calculated to evaluate the association between heart rate, AV delay and LV filling time. Interobserver and intraobserver reproducibility of the measurement of filling time at peak exercise was assessed using the coefficient of variation. $\mathrm{P}$ values less than 0.05 were 
considered significant. All analyses were performed with SPSS statistical package for Windows (SPSS 17.0, Chicago, Illinois). 


\section{Results}

\section{Phase 1 Study Population}

After meeting exclusion criteria ( $\mathrm{n}=58$ excluded), 83 patients were considered for recruitment into Phase 1 of the study. Of these, 11 patients declined, 9 patients were deemed too unstable to participate, 8 patients were unable to exercise and 3 had to be cancelled due to lack of manpower, leaving a final study population of 52 .

The baseline patient demographics and echocardiographic characteristics prior to implantation and at the time of optimization are shown in Table 2. At a median 6.0 (IQR 3.9-12.9 months) months following CRT the majority of patients were clinical responders to CRT, with an improvement in NYHA to class II in $78 \%$ and an improvement in mean ejection fraction to $40.2 \pm 12 \%$. Forty seven percent of patients demonstrated $\geq 15 \%$ reduction in LV end systolic volume post CRT. All patients were able to successfully complete the study protocol without complications. The LV lead was placed in a posterolateral $(n=19)$, posterior $(n=18)$, or lateral $(n=15)$ cardiac vein. The right atrial lead was positioned in the right atrial appendage and the right ventricular lead was placed in the septum $(n=36)$ or apex $(n=16)$ of the right ventricle.

\section{Optimization of the AV delay at rest}

The mean optimal AV delay obtained at rest was $114.2 \pm 29 \mathrm{~ms}$. 


\section{Optimization of the AV delay on exercise}

It was technically feasible to perform AV delay optimization at exercise in all study patients, with an interobserver and intraobserver coefficient of variation of LVFT measurement at peak exercise of $1.7 \%$ and $2.0 \%$ respectively. Moreover, there was a $100 \%$ interobserver concordance to within $20 \mathrm{~ms}$ of the final optimal AV delay on exercise. The resting heart rate $(\mathrm{HR})$ was $64.4 \pm 7.1 \mathrm{bpm}$, and although the majority of patients were taking prescribed negative chronotropic therapy, the HR increased to a peak heart rate of $103.0 \pm 13.1 \mathrm{bpm}$ at a mean exercise time of $7.1 \pm 2.3$ minutes. Optimal AV delay almost halved (49.5\% reduction) between rest and exercise (mean AV delay at rest $114 \pm 29.3 \mathrm{~ms}$, mean AV delay at peak exercise $57.3 \pm 31.5 \mathrm{~ms}$, mean difference $57.1 \mathrm{~ms}$, (95\% CI 65.2 to $48.7 \mathrm{~ms} \mathrm{p}<0.001$ ), (Figure 1). There was a corresponding increase in the mean LVFT from $241.2 \pm 65.2 \mathrm{~ms}$ to $280.6 \pm 7 \mathrm{~ms}$ following AV optimization at peak exercise $(\mathrm{p}<0.001)$. (Figure 2$)$ No patients required an increase in their resting optimal AV delay with exercise, and $3(6 \%)$ demonstrated no change from their optimal resting AV delay.

\section{Relationship of heart rate and LV filling time with AV delay.}

There was a linear association between the change in optimal AV delay from rest to exercise and the corresponding increase in the LVFT during exercise $(r=0.39 ; p=0.006)$ (Figure 3). A very weak but significant correlation was found between the degree of HR increase and the corresponding decrease in the optimal AV delay during exercise $(r=0.28$; $\mathrm{p}=0.047$, with the optimal AV delay reducing by a mean of $6.0 \mathrm{~ms}$ for every $10 \mathrm{bpm}$ of increase in HR with exercise. 


\section{Phase 2 -Cardiopulmonary exercise testing}

\section{Study Population}

Similar baseline characteristic were observed in the 20 patients as in the overall study population. All patients were in NYHA class I and II with a mean EF $42.5 \pm 11.7 \%$. Chronotropic medication included beta blockers in 18 patients (90\%) and digoxin in one patient (5\%). The mean optimal AV delay at rest was $115.5 \pm 29 \mathrm{~ms}$ with a mean optimal AV delay at peak exercise of $50.0 \pm 28.1 \mathrm{~ms}(\mathrm{p}<0.001)$

\section{Effect of rate adaptive AV delay $\mathrm{ON}$ versus $\mathrm{OFF}$}

\section{Heart rate}

There was no significant difference in baseline HR (RAAVD-OFF $69 \pm 10$ bpm versus RAAVD-ON $69 \pm 9$ bpm, p=0.6) or peak HR achieved $(123 \pm 20$ bpm for RAAVD-OFF versus $125 \pm 19 \mathrm{bpm}$ for RAAVD-ON, $\mathrm{p}=0.3$ ).

\section{Exercise time and VO2max}

Patients with RAAVD-ON achieved a significantly better exercise time $(8.7 \pm 3.2$ minutes) with a mean increase of 0.8 minutes $(95 \% \mathrm{CI}: 0.3$ to $1.2 ; \mathrm{p}=0.003)$ compared with RAAVD- OFF $(7.9 \pm 3.2$ minutes, Figure $4 a)$., There was a significant improvement in VO2max with RAAVD-ON $(14.9 \pm 3.7 \mathrm{ml} / \mathrm{kg} / \mathrm{min}$ for RAAVD-OFF and $16.1 \pm 4.0$ $\mathrm{ml} / \mathrm{kg} / \mathrm{min}$ for RAAVD-ON; $\mathrm{p}=0.024$, Figure $4 \mathrm{~b}$ ). Furthermore, 12 (60\%) patients achieved $\geq$ than $1 \mathrm{ml} / \mathrm{kg} /$ minute improvement in their VO2max with RAAVD-ON. However, no baseline predictors could identify those patients whose VO2max improved 
markedly (from the following variables: gender, age, hypertension, diabetes, ischemic etiology, echocardiographic response to CRT or device manufacturer). 


\section{Discussion}

This is the first study to report the effects of rate adaptive AV delay on exercise capacity in heart failure patients following CRT. Despite the variability in resting optimal AV delay, our results show a mean $50 \%$ shortening in the optimal AV interval with symptom limited exercise (mean difference $57.1 \mathrm{~ms}$ (95\% CI 65.2 to $48.7 \mathrm{~ms} \mathrm{p}<0.001)$. Rate adaptive AV delay translated into a $>15 \%$ increase in LVFT and almost $10 \%$ improvement in exercise capacity (as measured by peak VO2 and exercise duration), despite a lack of significant difference in peak HR achieved. These results are in keeping with earlier dual pacing studies which showed improved cardiac output and exercise tolerance with shorter AV intervals at rapid heart rates, and led to the development of rate adaptive AV delay programming $(3,4)$.

Normal physiological adaptation to exercise, under the effect of sympathetic stimulation and reduced vagal tone, leads to an increase in HR and a disproportionate shortening of diastole compared with systole, an effect more pronounced in patients with systolic heart failure due to longer systole at both rest and exercise (17). Diastolic duration is nonlinearly (inverse power) related to heart rate, shortening markedly for each $10 \mathrm{bpm}$ increase between 50 to $90 \mathrm{bpm}$ but becoming incrementally less pronounced at faster HRs (18). In contrast, isovolumic contraction time, systolic ejection time and isovolumic relaxation time demonstrate a linear negative correlation with increasing HR. (19). The cumulative effect is a marked reduction in diastolic filling time with increasing HR (20). 
Despite recent studies with negative findings with regard to AV delay optimization, such as Freedom (21) and SMART-AV (22) - our study adds the important dimension of exercise and underscores the strong dependence of optimal AV delay on heart rate. We propose that rate adaptive AV delay should be included in future studies on the benefit of AV optimization.

\section{The effect on the optimal AV delay during exercise.}

Forty seven of 50 patients evaluated (94\%) showed a significant reduction in optimal AV delay during exercise. Similar findings were reported by Sun et al (11) who showed an almost $40 \%$ reduction in the programmed AV delay during exercise, and Grimm et al (8), who observed a negative correlation between the optimal AV delay and HR in $80 \%$ of patients, with no patients requiring a reduction in AV delay with exercise, Both studies used the transmitral iterative method. . We have shown the feasibility and reproducibility of iterative $\mathrm{AV}$ delay optimization during exercise and use of this simple and robust method may account for the anticipated physiological shortening in the AV interval with exercise. This is in contrast to previously reported studies $(9,10)$ where a heterogeneous response to increasing HR had been observed. Scharf et al used non physiological atrial overdrive pacing to increase HR and pulsed-wave Doppler LVOT-VTI, in their CRT cohort and unexpectedly concluded that the AV delay should be prolonged at increasing HRs (5). This fuelled subsequent studies employing various different AV optimization methods, which have reported that the optimal AV delay could shorten, lengthen or remain constant with increasing HRs. 
Could the heterogeneous response to increasing HRs be attributed to the different AV delay optimization techniques and the different HR modulation methods used? Mokrani et al demonstrated a difference in the optimal AV delay at rest and on exercise depending on whether LVOT VTI or maximum LVFT was employed. (10) Using the iterative method, the exact mechanism that the operator is attempting to manipulate, (timing of ventricular filling) is viewed directly, rather than employing a surrogate outcome measure such as LVOT VTI, which is subject to significant variation due to small changes in the angle between the incident ultrasound beam and the outflow tract with respiration, an effect particularly exaggerated during exercise $(23,24)$. As the maximum improvement in cardiac output with AV delay optimization is in the region of $15-20 \%$ (1), any technique with a reported reproducibility of $4-10 \%$ during optimal resting conditions should be interpreted with caution during exercise, and may underlie the reported mixed results using these methods $(5,6,10)$.

\section{Is there a role for rate adaptive pacing?}

HF patients demonstrate blunting of the force-frequency relationship (FFR) due to rate dependent impairment of LV diastolic function and subsequent increase in LV end diastolic pressure (25). Manipulation of the LV filling pattern during exercise by AV optimization may augment the effective preload, thereby maintaining the stroke volume and enhancing exercise tolerance.

It is currently recommended that rate adaptive pacing should be switched OFF, $(7,10)$

however, rate adaptive AV delay (Medtronic Insynch models) was encouraged in the MIRACLE trials (26) and yet the order of clinical and remodelling response was similar 
to the other major CRT trials (EF improvement $4.6 \%$ at 6 months in MIRACLE, vs. 3.7\% at $3 \mathrm{~m}$ and $6.9 \%$ at $18 \mathrm{~m}$ in CARE-HF. Heart failure hospitalisations reduced by $45 \%$ in MIRACLE vs. $45 \%$ in CARE-HF). In this study, rate adaptive AV delay translated into a 48 second increase in exercise time and a $>1.0 \mathrm{ml} / \mathrm{kg} /$ minute increase VO2 max. This magnitude of improvement in VO2 max, although not apparently large, is similar to the magnitude of change seen with CRT in the major trials (CRT $1.05 \mathrm{ml} / \mathrm{kg} / \mathrm{min}$ vs. Control $0.39 \mathrm{ml} / \mathrm{kg} / \mathrm{min}$ with weighted mean difference of $0.65 \mathrm{ml} / \mathrm{kg} /$ minute, $95 \% \mathrm{CI} 0.27-1.04$ ).

\section{Clinical significance}

Replication of these findings in larger studies may lead to the development of an integrated, dynamic pacemaker algorithm, providing additional functional benefits to ambulant HF patients implanted with CRT devices. Furthermore, the rate adaptive AV algorithms of the different devices (particularly Medtronic and Biotronik) will need to provide more flexibility in their settings so as to allow for the full reduction to the shortest optimal AV delay at peak exercise, which currently is not achievable with existing device settings.

\section{Limitations}

As this is a pilot study, only a small number of patients were included in the Phase 2 CPET trial. 
Secondly, only $16 \%$ of patients in our study were in NYHA class 3 , and the majority were in NYHA 2 or below, hence our study conclusions may not be relevant to patients in NYHA 3.

Thirdly, participants included in the study were implanted with different manufacturer models of devices, each with their unique rate adaptive AV delay algorithm. This may have impacted on the results of the Phase 2 study, but does reflect real life clinical practice.

Fourthly, Phase 2 of the study included atrial sensed patients in sinus rhythm only, so the findings cannot be extrapolated to the entire CRT population.

Finally, the short term nature of our study does not provide information about the long term outcome of AV optimization during exercise. However, the inclusion of VO2max, a useful prognostic marker in HF patients (28), as a clinical outcome in the study, may provide some clues to the possible translation to long term prognosis.

\section{Conclusion}

Despite current contrary recommendations, this study showed that programming a rate adaptive AV delay translated into improvement in exercise capacity in HF CRT patients. The debate regarding rate adaptive AV delay in the CRT population should now be revisited and further studies are required to confirm our findings. Given the current inter vendor protocol variations, the best algorithm will also need to be established. 
Acknowledgments. The authors thank the Bioengineering Department of St George's Healthcare NHS Trust for their expert assistance in the modification of the Tenturi E80 exercise bicycle. 


\section{References}

(1) Auricchio A, Stellbrink C, Block M et al. Effect of pacing chamber and atrioventricular delay on acute systolic function of paced patients with congestive heart failure. The Pacing Therapies for Congestive Heart Failure Study Group. The Guidant Congestive Heart Failure Research Group. Circulation 1999 June 15;99(23):2993-3001.

(2) Daubert C, Ritter P, Mabo P, Ollitrault J, Descaves C, Gouffault J. Physiological relationship between AV interval and heart rate in healthy subjects: applications to dual chamber pacing. Pacing Clin Electrophysiol 1986 November; 9(6 Pt 2):1032-9.

(3) Ritter P, Daubert C, Mabo P, Descaves C, Gouffault J. Haemodynamic benefit of a rate-adapted A-V delay in dual chamber pacing. Eur Heart J 1989 July;10(7):637-46.

(4) Melzer C, Korber T, Theres H, Nienaber CA, Baumann G, Ismer B. How can the rateadaptive atrioventricular delay be programmed in atrioventricular block pacing? Europace 2007 May;9(5):319-24.

(5) Scharf C, Li P, Muntwyler J et al. Rate-dependent AV delay optimization in cardiac resynchronization therapy. Pacing Clin Electrophysiol 2005 April;28(4):279-84

(6) Valzania C, Eriksson MJ, Boriani G, Gadler F. Cardiac resynchronization therapy during rest and exercise: comparison of two optimization methods. Europace 2008 October;10(10):1161-9. 
(7) Melzer C, Bondke H, Korber T, Nienaber CA, Baumann G, Ismer B. Should we use the rate-adaptive AV delay in cardiac resynchronization therapy-pacing? Europace 2008 January; 10(1):53-8.

(8) Grimm RA, Sun JP, Agler D et al.. Shorter AV delays provide improved echocardiographic hemodynamics during exercise in patients receiving cardiac resynchronization therapy. Pacing Clin Electrophysiol 2009 April;32(4):457-65.

(9) Whinnett ZI, Briscoe C, Davies JE et al. The atrioventricular delay of cardiac resynchronization can be optimized hemodynamically during exercise and predicted from resting measurements. Heart Rhythm 2008 March;5(3):378-86.

(10) Mokrani B, Lafitte S, Deplagne A, et al. Echocardiographic study of the optimal atrioventricular delay at rest and during exercise in recipients of cardiac resynchronization therapy systems. Heart Rhythm 2009 July;6(7):972-7.

(11) Sun JP, Lee AP, Grimm RA et al. Optimisation of atrioventricular delay during exercise improves cardiac output in patients stabilised with cardiac resynchronisation therapy. Heart. 2011 August doi:10.1136/heartjnl-2011-300278

(12) Jansen AH, Bracke FA, van Dantzig JM et al. Correlation of echo-Doppler optimization of atrioventricular delay in cardiac resynchronization therapy with invasive 
hemodynamics in patients with heart failure secondary to ischemic or idiopathic dilated cardiomyopathy. Am J Cardiol 2006; 97(4):552-557.

(13) Eberhardt F, Hanke T, Fitschen J et al. AV interval optimization using pressure volume loops in dual chamber pacemaker patients with maintained systolic left ventricular function. Clin Res Cardiol. 2012 Mar 9.

(14) Gorcsan J, III, Abraham T, Agler DA et al. Echocardiography for cardiac resynchronization therapy: recommendations for performance and reporting--a report from the American Society of Echocardiography Dyssynchrony Writing Group endorsed by the Heart Rhythm Society. J Am Soc Echocardiogr 2008 March;21(3):191-213.

(15) Cleland JG, Daubert JC, Erdmann E et al. The CARE-HF study (CArdiac REsynchronisation in Heart Failure study): rationale, design and end-points. Eur J Heart Fail 2001 August;3(4):481-9.

(16) Lee L, Campbell R, Scheuermann-Freestone M et al. Metabolic modulation with perhexiline in chronic heart failure: a randomized, controlled trial of short-term use of a novel treatment. Circulation 2005 November 22;112(21):3280-8.

(17) Plehn G, Vormbrock J, Perings C et al.. Loss of diastolic time as a mechanism of exercise-induced diastolic dysfunction in dilated cardiomyopathy. Am Heart J 2008 June;155(6):1013-9. 
(18) Barbieri D, Percoco GF, Toselli T, Guardigli G, Ansani L, Antonioli GE. AV delay and exercise stress tests: behavior in normal subjects. Pacing Clin Electrophysiol. 1990 Dec;13(12 Pt 2):1724-7

(19) Cui W, Roberson DA, Chen Z, Madronero LF, Cuneo BF. Systolic and diastolic time intervals measured from Doppler tissue imaging: normal values and Z-score tables, and effects of age, heart rate, and body surface area. J Am Soc Echocardiogr 2008 April;21(4):361-70.

(20) Chung CS, Karamanoglu M, Kovacs SJ. Duration of diastole and its phases as a function of heart rate during supine bicycle exercise. Am J Physiol Heart Circ Physiol 2004 November;287(5):H2003-H2008.

(21) Abraham WT, Gras D, Yu CM et al. Results from the Freedom Trial - Assess the safety and efficacy of frequent optimization of cardiac resynchronization therapy. (abstract). http://www.hrsonline.org/Sessions/ScientificProgram/upload/Results-FromThe-Freedom-Trial-Assess-The-Safety-And-Efficacy-Of-Frequent-Optimisation-OfCardiac-Resynchronisation-Therapy.pdf

(22) Ellenbogen KA, Gold MR, Meyer TE et al. Primary Results From the SmartDelay Determined AV Optimization: A Comparison to Other AV Delay Methods Used in Cardiac Resynchronization Therapy (SMART-AV) Trial: A Randomized Trial Comparing Empirical, Echocardiography-Guided, and Algorithmic Atrioventricular 
Delay Programming in Cardiac Resynchronization Therapy. Circulation. 2010; 122:26608.

(23) Turcott RG, Witteles RM, Wang PJ, Vagelos RH, Fowler MB, Ashley EA. Measurement precision in the optimization of cardiac resynchronization therapy. Circ Heart Fail 2010 May;3(3):395-404.

(24) Pabari PA, Willson K, Stegemann B et al. When is an optimization not an optimization? Evaluation of clinical implications of information content (signal-to-noise ratio) in optimization of cardiac resynchronization therapy, and how to measure and maximize it. Heart Fail Rev 2011 May;16(3):277-90.

(25) Vollmann D, Luthje L, Schott P, Hasenfuss G, Unterberg-Buchwald C. Biventricular pacing improves the blunted force-frequency relation present during univentricular pacing in patients with heart failure and conduction delay. Circulation 2006 February 21;113(7):953-9.

(26) Abraham WT, Fisher WG, Smith AL et al. MIRACLE Study Group. Multicenter InSync Randomized Clinical Evaluation. Cardiac resynchronization in chronic heart failure. N Engl J Med. 2002 Jun 13;346(24):1845-53.

(27) McAlister F, Ezekowitz J, Wiebe $\mathrm{N}$ et al. Cardiac resynchronization therapy for congestive heart failure. Evid Rep Technol Assess (Summ). 2004;106:1-8. 
(28) Mancini DM, Eisen H, Kussmaul W, Mull R, Edmunds LH, Jr., Wilson JR. Value of peak exercise oxygen consumption for optimal timing of cardiac transplantation in ambulatory patients with heart failure. Circulation 1991 March;83(3):778-86. 
Table 1. Standard algorithms for rate adaptive AV delay for the three models in Phase 2.

\begin{tabular}{|c|c|}
\hline CRT- ICD or CRT model & $\begin{array}{c}\text { Rate adaptive AV delay } \\
\text { protocol }\end{array}$ \\
\hline Guidant Contak Renewal TR2 $($ CRT-P) & $\begin{array}{c}\text { Steps of 2ms between maximal and } \\
\text { minimal AV delay }\end{array}$ \\
\hline Biotronik Lumax 540 HF-T® (CRT-ICD) & $\begin{array}{c}5 \mathrm{~ms} \text { AV delay decrease per } 10 \mathrm{bpm} \\
\text { between } 50 \text { and } 150 \mathrm{bpm}\end{array}$ \\
\hline $\begin{array}{c}\text { Medtronic Concerto II D294TRK® (CRT- } \\
\text { ICD) }\end{array}$ & $\begin{array}{c}\text { 10ms AV delay decrease per } 10 \mathrm{bpm} \\
\text { between } 80 \text { and } 120 \mathrm{bpm}\end{array}$ \\
\hline
\end{tabular}


Table 2: Patient Characteristics for all 52 patients in Phase 1 of the study.

\begin{tabular}{|c|c|}
\hline $\begin{array}{c}\text { Variable } \\
\text { Characteristics }\end{array}$ & Phase 1. $(\mathrm{N}=52)$ \\
\hline Age, years & $71.6 \pm 9.2$ \\
\hline Gender (female), n (\%) & $13(25.0)$ \\
\hline Hypertension, n (\%) & $28(53.8)$ \\
\hline Diabetes, n (\%) & $15(28.8)$ \\
\hline Ischemic etiology, n (\%) & $29(55.8)$ \\
\hline CRT-P, n (\%) & $36(69.2)$ \\
\hline \multicolumn{2}{|l|}{ Pre CRT-Characteristics } \\
\hline \multicolumn{2}{|l|}{ NYHA } \\
\hline III, n (\%) & $37(71.2)$ \\
\hline $\mathrm{IV}, \mathrm{n}(\%)$ & $15(28.8)$ \\
\hline QRS, ms & $159.8 \pm 23.1$ \\
\hline $\mathrm{PR}, \mathrm{ms}$ & $214.3 \pm 37.6$ \\
\hline $\mathrm{EF},(\%)$ & $30.2 \pm 6.5$ \\
\hline LVEDV, $\mathrm{ml}$ & $221.5 \pm 56.3$ \\
\hline LVESV, ml & $163.4 \pm 54.0$ \\
\hline \multicolumn{2}{|l|}{ Post CRT-characteristics } \\
\hline \multicolumn{2}{|l|}{ NYHA } \\
\hline $\mathrm{I}, \mathrm{n}(\%)$ & $3(6.0)$ \\
\hline II, n (\%) & $39(78.0)$ \\
\hline III, n (\%) & $8(16.0)$ \\
\hline $\mathrm{EF},(\%)$ & $40.2 \pm 11.7$ \\
\hline LVEDV, ml & $177.9 \pm 68.0$ \\
\hline LVESV, ml & $119.6 \pm 63.8$ \\
\hline Clinical responder, $\mathrm{n}(\%)^{*}$ & $42(82.4)$ \\
\hline Echo responder, $\mathrm{n}(\%)$ & $24(47.1)$ \\
\hline \multicolumn{2}{|l|}{ Treatments } \\
\hline Beta blockers, n (\%) & $47(92.2)$ \\
\hline ACEI n (\%) & $48(94.1)$ \\
\hline Spironolactone, n (\%) & $15(29.4)$ \\
\hline Furosemide, n (\%) & $46(90.2)$ \\
\hline Amiodarone, n (\%) & $5(9.8)$ \\
\hline Digoxin, $\mathrm{n}(\%)$ & $2(3.8)$ \\
\hline
\end{tabular}


Data are presented as the mean value $\pm \mathrm{SD}$ for continuous variables and number and percentage of patients for nominal data.

CRT-P $=$ cardiac resynchronization therapy- biventricular pacing only; ACEI= angiotensin converting enzyme inhibitor; $\mathrm{EF}=$ ejection fraction NYHA= New York Heart Association; LVEDD= left ventricular end diastolic volume; LVESD= left ventricular end systolic volume.

* Clinical responder defined as $\geq 1$ reduction in NYHA class post CRT.

Echo responder defined as patients exhibiting $\geq 15 \%$ reduction in LVESV post CRT. 


\section{Figure Legends}

Figure 1: Change in optimal AV delay assessed at rest and peak exercise, $n=52$ subjects.

Figure 2: The mean left ventricular filling time (ms) prior to and following AV optimization at rest and during exercise in the 52 study patients.

Figure 3: Linear regression analyses correlating left ventricular filling time and change in optimal AV delay during exercise optimisation.

Figure 4: Comparison for (A) exercise duration and (B) peak myocardial oxygen consumption are shown for rate adaptive pacing programmed ON versus OFF during cardiopulmonary exercise testing for the 20 study patients.

VO2 $\max =$ peak myocardial oxygen consumption 
Figure 1.

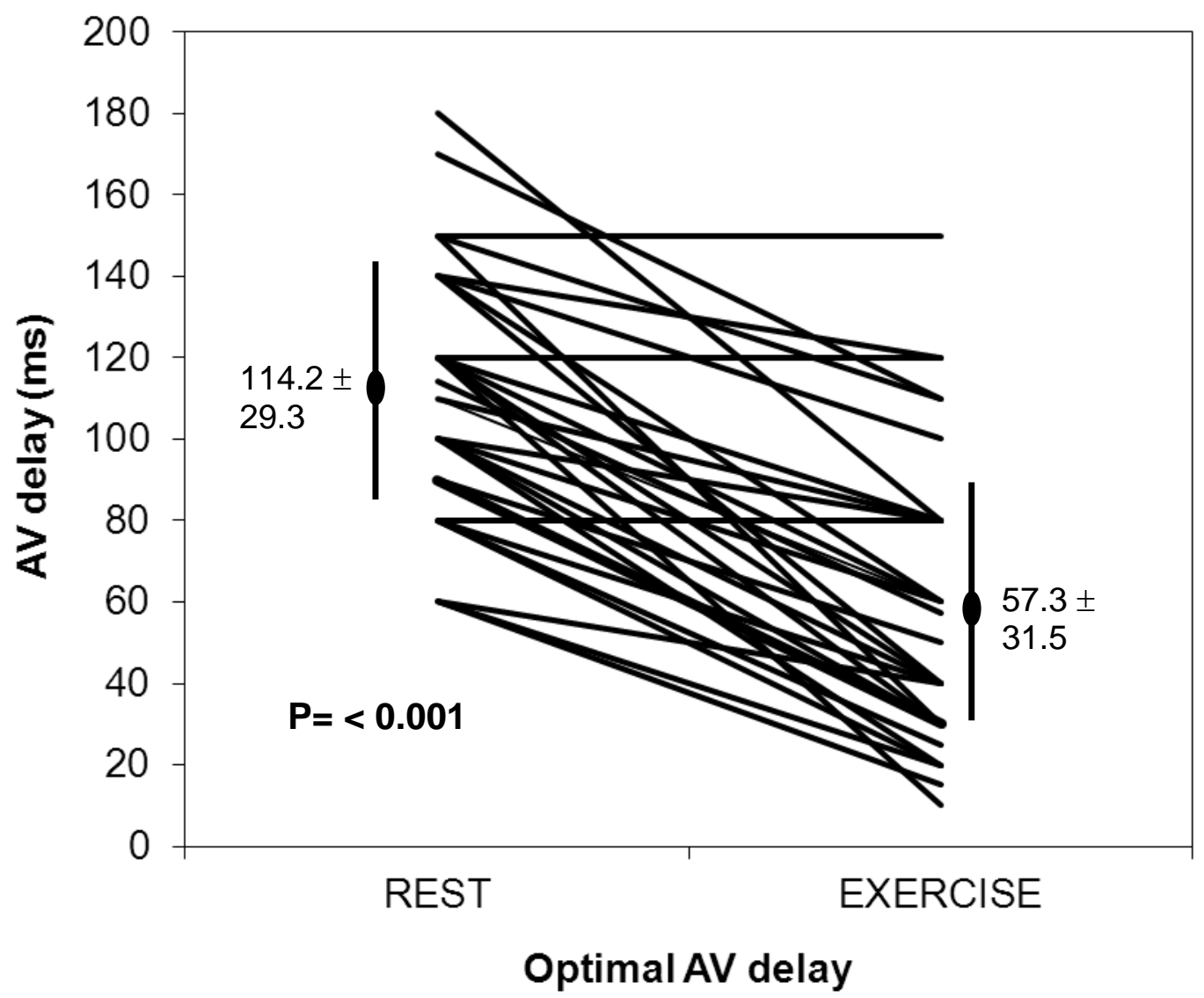


Figure 2.

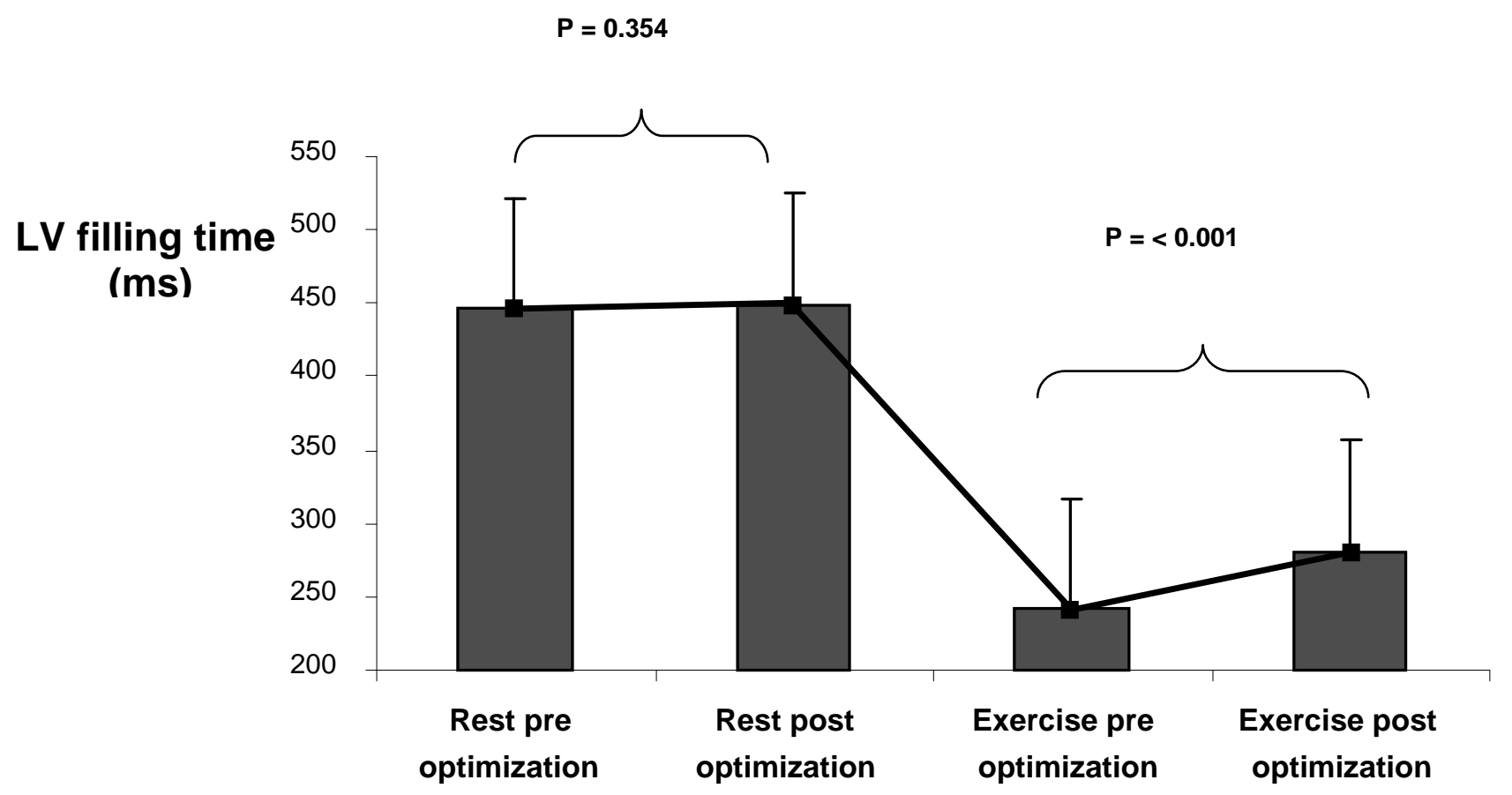


Figure 3.

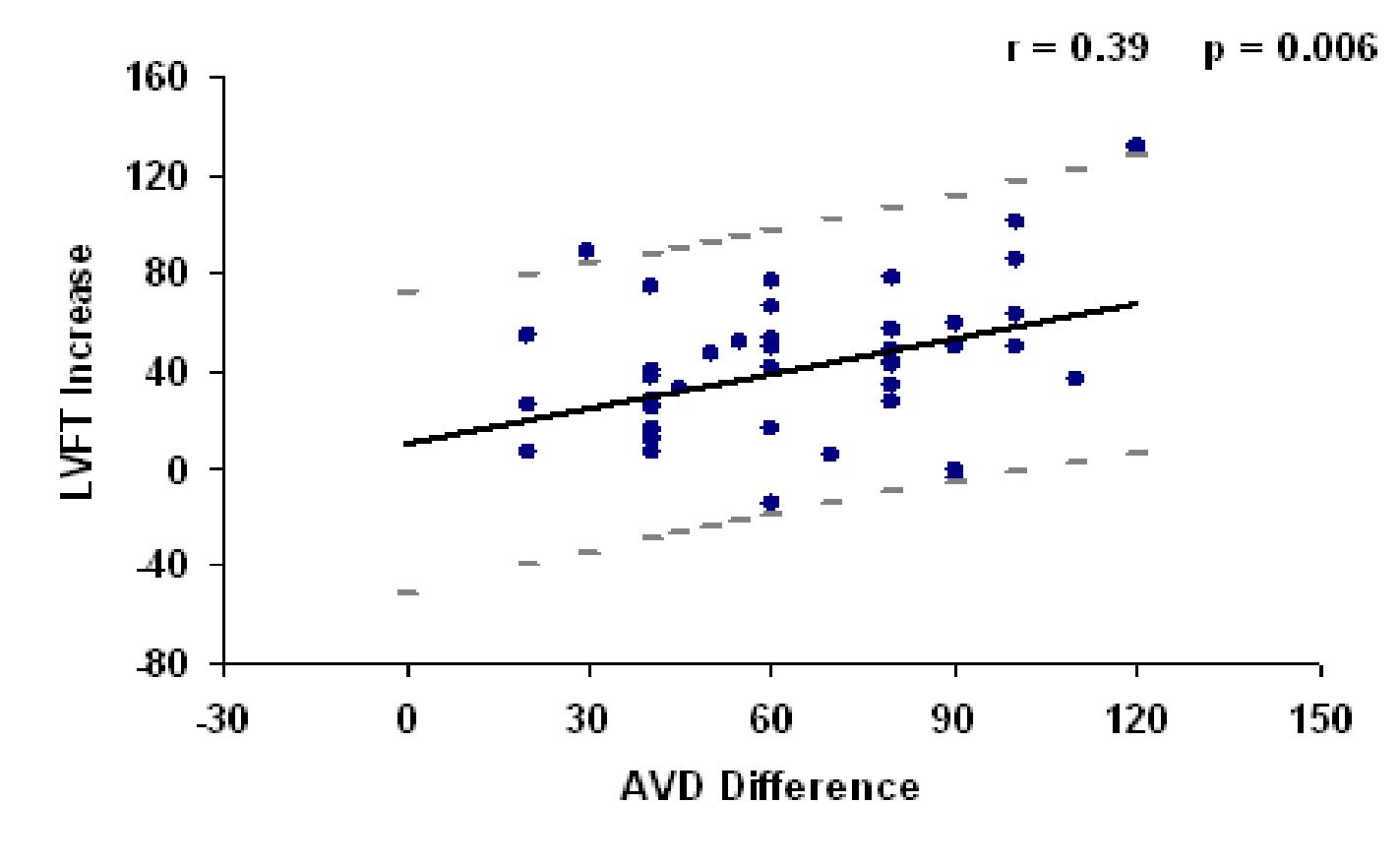


Figure 4A.

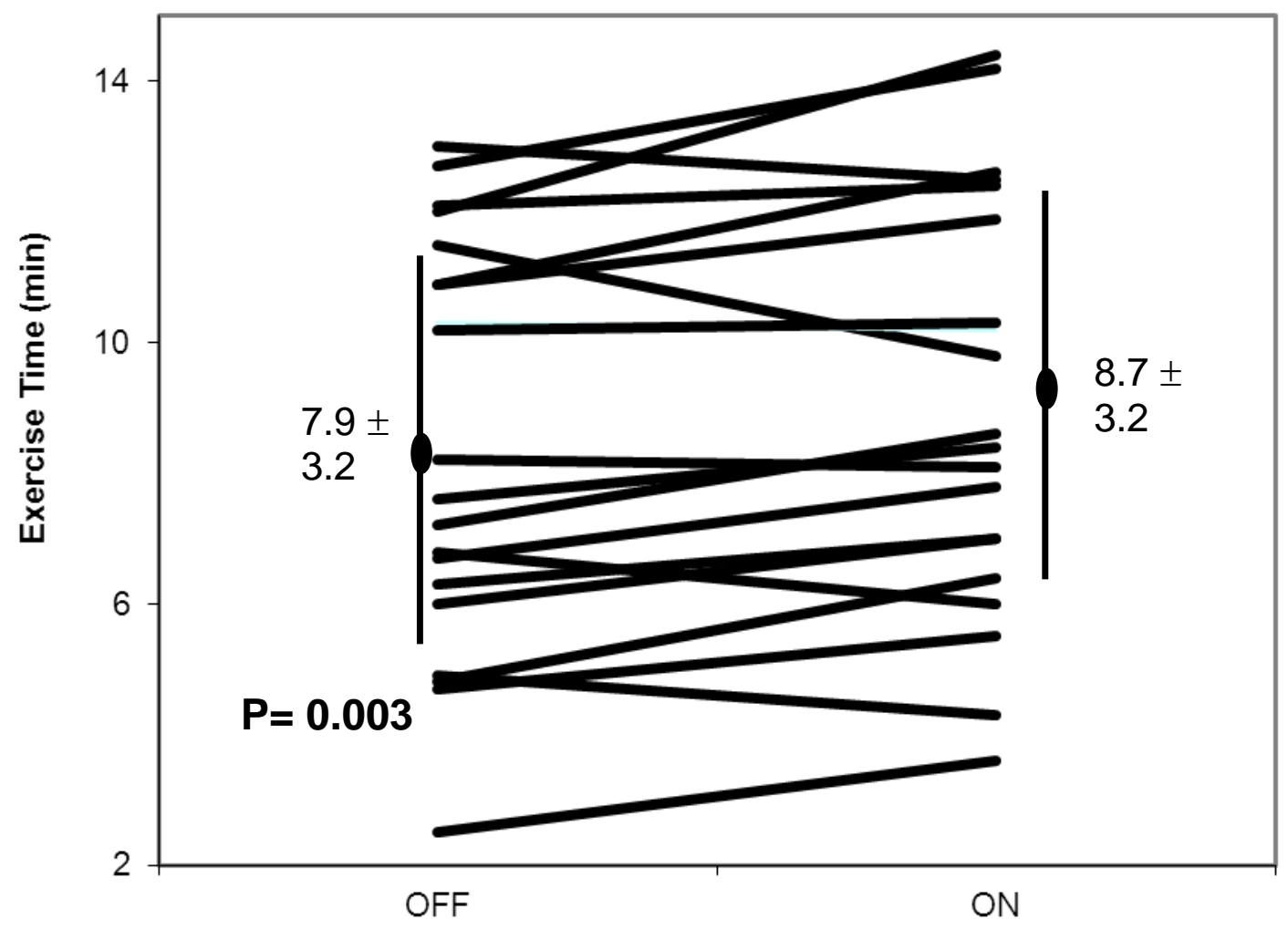

Programmable Rate Adaptive AV Delay 
Figure 4B.

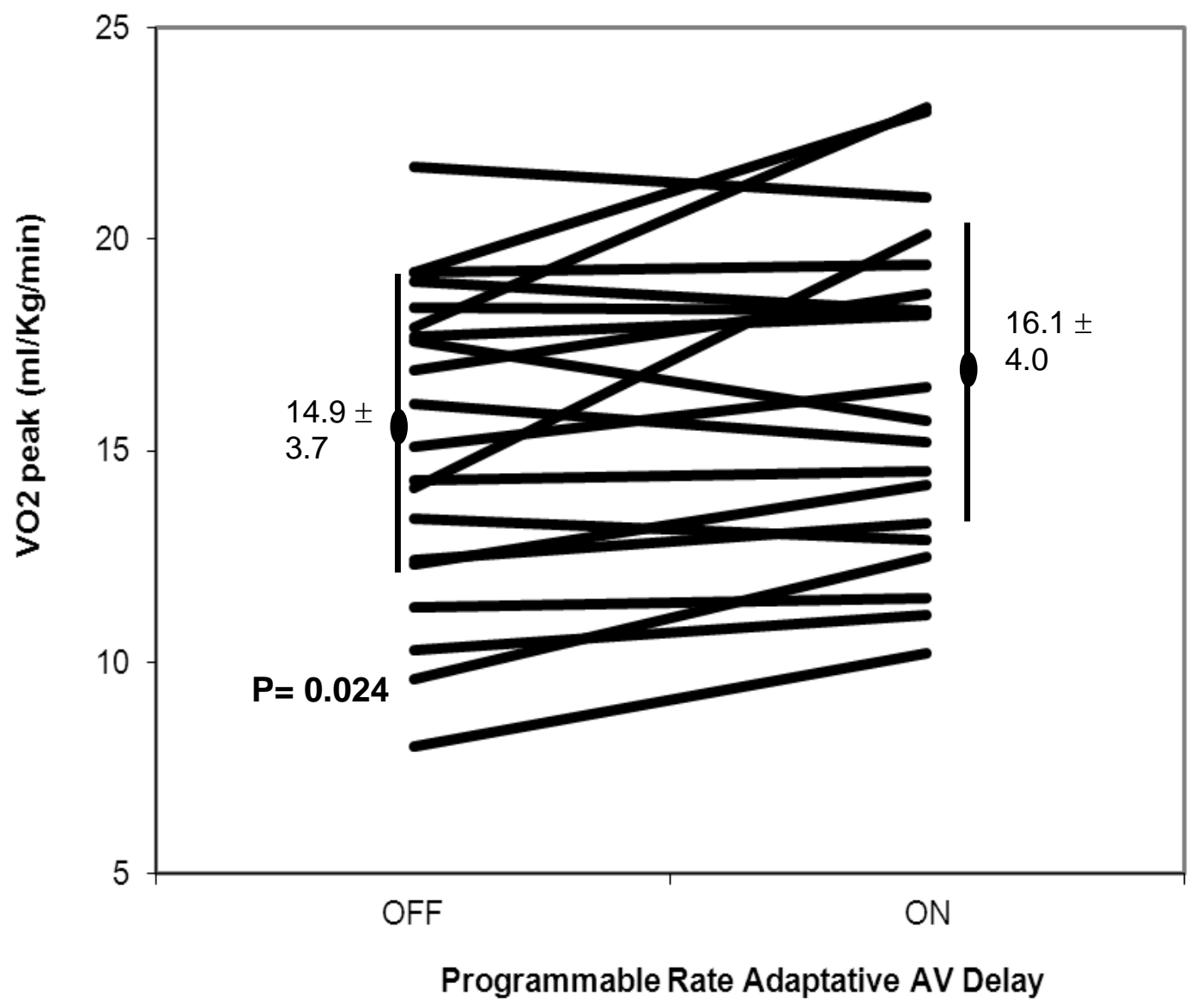

